\title{
Commissioning of a beam screen test bench experiment with a future circular hadron collider type synchrotron radiation beam
}

\author{
L. A. Gonzalez $\odot^{*}$ \\ CERN, The European Organization for Nuclear Research, CH-1211 Geneva, Switzerland \\ and LNF-INFN, Via Enrico Fermi, 4000044 Frascati, Roma, Italy \\ M. Gil-Costa \\ CIEMAT, Madrid, 28040 Spain \\ and CERN, The European Organization for Nuclear Research, CH-1211 Geneva, Switzerland \\ V. Baglin, P. Chiggiato, C. Garion, and R. Kersevan \\ CERN, The European Organization for Nuclear Research, CH-1211 Geneva, Switzerland \\ S. Casalbuoni and E. Huttel \\ KARA, Karlsruhe Institute of Technology, P.O. Box 3640, D-76021 Karlsruhe, Germany \\ I. Bellafont and F. Perez \\ ALBA Synchrotron Light Source, Barcelona, 08290 Spain
}

(Received 2 April 2019; published 23 August 2019)

\begin{abstract}
Coping with synchrotron radiation (SR) that originated at superconducting bending magnets is one of the major challenges in the design of the vacuum beam pipes of hadron colliders. In the case of the Future Circular hadron Collider (FCC-hh), similarly as for the LHC, a beam screen, operating at higher temperatures than the cold mass, has been designed in order to preserve the superconducting magnet cold bores from direct synchrotron irradiation. The quality of the beam screen vacuum can be severely compromised by the absorption of SR into its walls, enhancing the risk for numerous beam detrimental effects to arise. In order to experimentally study such effects and develop strategies for their minimization, a beam screen test bench experiment (BESTEX) has been conceived and installed in the Karlsruhe Research Accelerator storage ring at the Karlsruhe Institute for Technology. The BESTEX has been designed to explore photon stimulated desorption, photon reflectivity, photon heat loads, and photoelectron generation originated on beam screen prototypes under irradiation of the FCC-hh-like SR spectrum. A detailed description of the BESTEX, its commissioning, and its functionality is hereby presented.
\end{abstract}

DOI: 10.1103/PhysRevAccelBeams.22.083201

\section{INTRODUCTION}

Synchrotron radiation (SR) is known to be at the origin of numerous detrimental beam effects in particle accelerators [1]. The severity of these SR countereffects is significantly enhanced when considering the construction of machines beyond the cutting edge of collision energies such as the Future Circular hadron Collider (FCC-hh). The FCC-hh, studied as a successor of the Large Hadron Collider (LHC), aims to expand the current energy frontiers, providing hadron collisions at a center of mass of

\footnotetext{
*luis.gonzalez.gomez@cern.ch

Published by the American Physical Society under the terms of the Creative Commons Attribution 4.0 International license. Further distribution of this work must maintain attribution to the author(s) and the published article's title, journal citation, and DOI.
}

$100 \mathrm{TeV}$ [2-4]. During its operation, proton beams stored in the FCC-hh's arcs would originate unprecedented levels of SR for hadron machines [4-7]. Under these conditions, the limits at which the FCC-hh can operate are determined by the capabilities of minimizing SR-related effects, such as heat load, photon stimulated desorption (PSD), and photoelectron generation.

As in the case of the LHC [8,9], big efforts have been put into the design of the FCC-hh beam screen (BS) [6,10], which is essential to protect the cold superconducting magnets from the direct irradiation high-power SR photon beams. The validation of the current FCC-hh BS design requires one to analyze the response of the corresponding $\mathrm{BS}$ prototypes under significant levels of SR. To that end, in order to explore the vacuum performance of the FCC-hh BS, a beam screen test bench experiment (BESTEX) was designed and built at CERN and installed in the $2.5 \mathrm{GeV}$ electron storage ring Karlsruhe Research Accelerator 


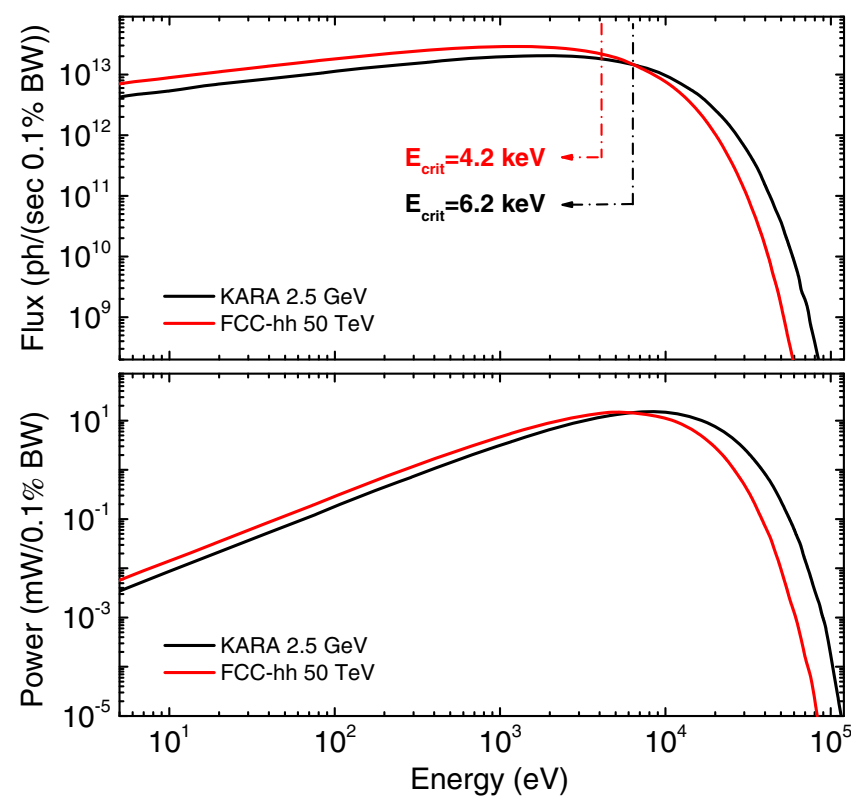

FIG. 1. Comparison of the photon flux (a) and power (b) of the FCC-hh at $50 \mathrm{TeV}$ proton beam energy in comparison with KARA's at its nominal $2.5 \mathrm{GeV}$. Calculations were performed with SYNRAD+.

(KARA) light source at the Karlsruhe Institute of Technology (KIT). The BESTEX aims to explore the photon stimulated desorption phenomena, as well as photon reflectivity, photoelectron generation, and heat loads during irradiation of the FCC-hh BS. KARA has been chosen due to its similarities with the FCC-hh in terms of SR spectrum, photon flux, and power (see Fig. 1). Because of the similarities between KARA and FCC-hh's SR spectra and critical energies (defined as the energy at which the SR power spectrum is divided in two equal parts), the simulation techniques used to estimate the response of the samples tested in the BESTEX can also be applied to the actual FCC-hh BS during machine operation.

\section{THE SETUP}

The BESTEX is an experimental instrument that allows one to study SR-induced effects on non-leak-tight BSs, at room and cryogenic temperatures, under ultrahigh vacuum (UHV) conditions. The BESTEX's schematic layout is presented in Fig. 2. The samples under study are typically 2-m-long FCC-hh BS prototypes (see Sec. V), which are placed and aligned (see Sec. IV) inside the setup. The setup is an UHV system formed by two stages, namely, the front end and test bench. The front end has the function to allow the extraction of the synchrotron light into the test bench. A sector gate valve allows one to isolate the test bench from the vacuum of KARA, which permits one to install and exchange the samples without breaking the vacuum integrity of the storage ring. A perforated crotch absorber (20 mm opening), located upstream from the front end, crops the photon fan generated at the bending magnet and defines the maximum SR flux and power allowed into the test bench, i.e., $180 \mathrm{~W}$ and $5.2 \times 10^{17} \mathrm{ph} / \mathrm{s}$, respectively, for a $2.5 \mathrm{GeV}$ and $130 \mathrm{~mA} e^{-}$beam. On the other hand, a pneumatically actuated sector photon absorber is hosted immediately after the crotch absorber, allowing one to intercept the totality of the photons passing through its aperture. This permits one to connect (when necessary) both KARA's and BESTEX's vacua while keeping the system free of irradiation. The front end is also equipped with a collimating stage, placed after the sector valve, at a distance of $3550 \mathrm{~mm}$ from the photon source at KARA's bending magnet. It is composed of a total of four slits, arranged in pairs, which can be independently displaced. Each pair of slits can move in the horizontal and the vertical direction, respectively, in a plane perpendicular to the SR axis. This allows the SR fan generated at KARA's dipole magnet to be cropped into a beam of rectangular shape, enabling one to control the size, flux, and power of the photon beam that irradiates the sample under study. After the collimator stage, the photons travel a distance of $\sim 1800 \mathrm{~mm}$ (depending on the angle of incidence) before impinging in the center of the sample. A fluorescent screen, described below, is also housed downstream after the slits for SR visualization, through an UHV view port, via a fixed video camera installed outside the setup. The whole test bench lays on two separated supports. Each support is equipped with a motorized stage, enabling their independent movement in the direction perpendicular to the incoming photon beam. In this way, the test bench can be oriented with respect to the direction of the photon beam. By doing so, the inner walls of the sample under study are irradiated with a glancing angle given by the position of the test bench as depicted in Fig. 2. By modifying the position of the setup, the orientation of the sample with respect to the photon beam can be chosen, allowing one to irradiate at angles down to $2 \mathrm{mrad}$. The collimator's opening and the orientation of the test bench can be chosen so as to irradiate the sample under study at various possible glancing angles.

The front end and the test bench are connected by an edge-welded bellows, which allows the necessary lateral displacement to move the test bench with respect to the fixed front end assembly. The experimental diagnostics equipment is distributed in four assemblies placed along the test bench, i.e., the (i) back instrumentation assembly (BIA), located at the back end of the setup, (ii) front instrumentation assembly (FIA), immediately after the front end, (iii) sample chamber assembly (SCA), placed in between the BIA and FIA, and (iv) middle instrumentation assembly (MIA), housed at the middle point of the SCA.

The SCA is a 1.8-m-long tubular UHV chamber, of $100 \mathrm{~mm}$ inner diameter, in which the sample under study is installed and fiducialized. The extremities of the sample 


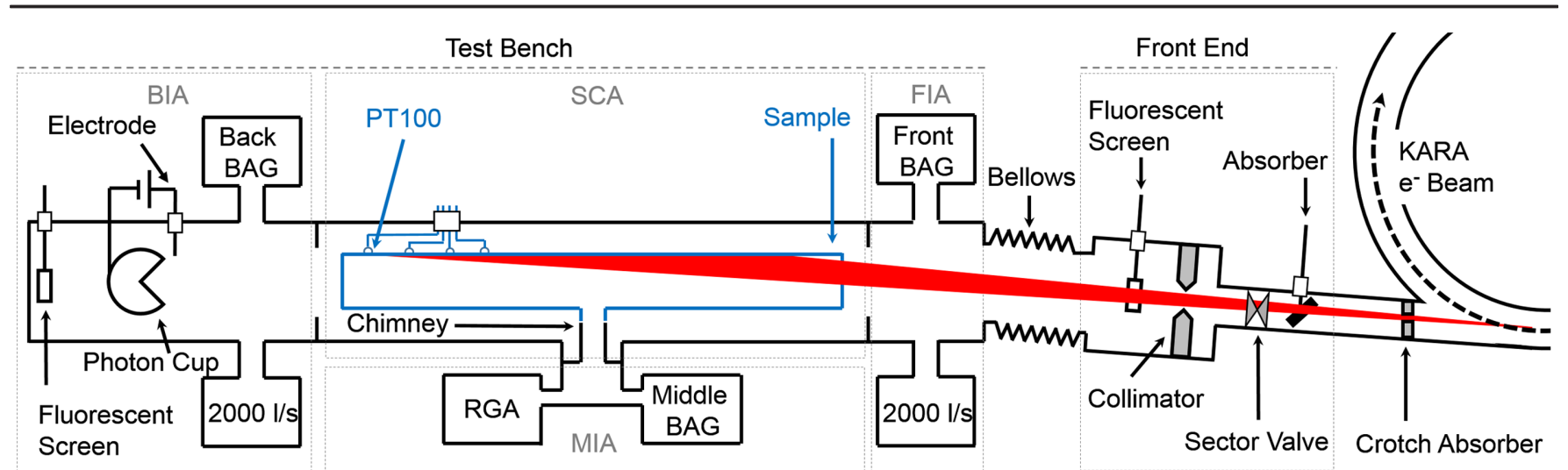

FIG. 2. Schematic layout of the BESTEX.

( 2 m long) protrudes into the FIA and MIA at each side. Placed along the axial direction of the SCA, there are five DN63 vacuum ports, on which the necessary electrical feedthroughs are housed. Two extra DN63 ports are present at the chamber's middle point. One of them, placed at the bottom side of the SCA chamber, is used as a connection port to the MIA. Another one, placed on the top of the SCA chamber, hosts a pin used to maintain the sample in the desired position, avoiding its tilt around the axial direction. A ceramic piece is used to thermally isolate the pin from the sample. Located at each extremity, the SCA has two sets of three DN16 vacuum flanges, as depicted in Fig. 3. The latter hosts six UHV linear actuators, which hold the sample inside the vacuum vessel and allow the positioning of the sample in its designated orientation with respect to the chamber's axis. Each of the linear actuators is equipped with a ceramic cap, which thermally isolates the sample from the rest of the setup. A total of three calibrated Bayard-Alpert gauges (BAGs) are installed on the BIA, FIA, and MIA, named as B-BAG, F-BAG, and M-BAG, respectively. The pressure

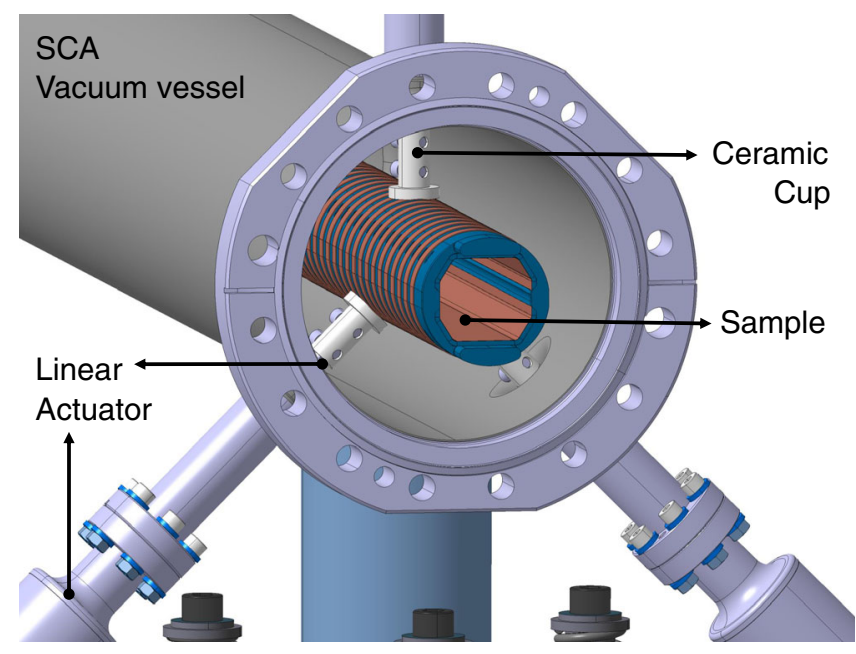

FIG. 3. 3D model of the linear actuators used to hold the samples inside the UHV chamber, installed at one of the extremities of the SCA at the BESTEX. readings of the gauges are expressed in mbar nitrogen equivalent $\left(\mathrm{N}_{e q}\right)$. The MIA also hosts a calibrated residual gas analyzer (RGA) that allows one to perform analyses of the residual gas species during sample irradiation. Both the FIA and BIA are equipped with a $2000 \mathrm{l}$ /s NEG cartridge pump. Each of them is equipped with an auxiliary $10 \mathrm{l} / \mathrm{s}$ ion pump for $\mathrm{CH}_{4}$ pumping. The pumps, as well as B-BAG and F-BAG, are installed at equal distances from the middle point of the SCA. Such a configuration ensures a symmetric pressure distribution along the sample and enables the monitoring of the pressure profile along the test bench, including the parts of the setup aside from the sample under study. A primary pumping system is attached to the FIA and can be isolated by an UHV all-metal valve once the NEG pump cartridges are activated.

The BESTEX has two water-cooled fluorescent screens [see the 3D model in Fig. 4(a)] housed at the BIA and front

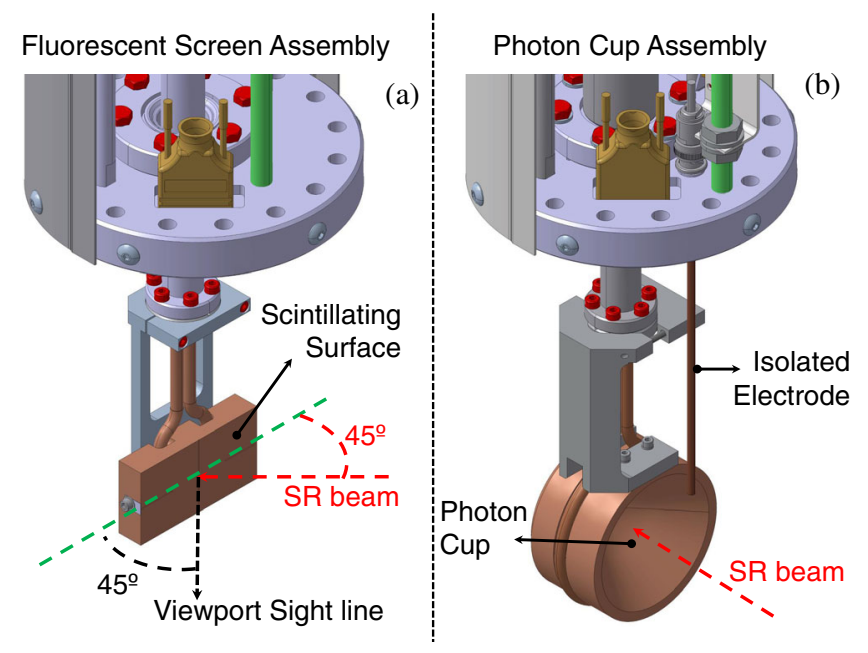

FIG. 4. 3D models of (a) a fluorescent screen assembly-the dashed arrows represents the geometry of the SR beam and the view port sight line with respect to the screen's paddle; in both figures, the red arrow represents the direction of the sample's longitudinal axis - and (b) the photon cup assembly of the BESTEX, where the conical OFHC bulk and the isolated clearing electrode can be observed. 
end (B-FLUO and F-FLUO, respectively) and separated by a distance of $3 \mathrm{~m}$. Each of them consists of an oxygenfree high thermal conductivity $\mathrm{Cu}(\mathrm{OFHC})$ block and is designed to accept the irradiation of the incoming SR beam. The front plate of each fluorescent screen $(70 \times 40 \mathrm{~mm})$ is coated with a $100-\mu \mathrm{m}$-thick scintillating layer of yttrium oxide europium phosphor [11], thereby allowing one to monitor the SR beam's footprint and, when necessary, to deploy the footprints generated by the visible light and the $\mathrm{x}$ rays. The irradiated surface is visible through a view port placed at each corresponding vessel. The screen paddles are aligned at an angle of $45^{\circ}$ with respect to the longitudinal axis of the setup and the line of sight of each view port. A camera is fitted to each view port in order to enable remote visualization of the photon beam during operation. F-FLUO is irradiated by the photons immediately after passing through the collimating slits. A pneumatic actuator allows its movement in the vertical direction so that the screen can be set to intercept the photon beam only when position monitoring is necessary. At the opposite end, B-FLUO is fixed and collects all the photons reaching the very end of the test bench. The information of both fluorescent screens is then analyzed together to determine the photon beam trajectory along the setup. This becomes specially critical when considering the vertical tilts in the photon beam trajectory due to the possible oscillations of the $e$ beam when passing through KARA's bending magnets. The fluorescent screens have $5 \mathrm{~mm}$ horizontal and $2 \mathrm{~mm}$ vertical grid lines, machined on the copper block in order to reference the position of the photon beams. According to the size of the grid cells, the visual inspection of the SR beam footprint allows one to verify the photon beam trajectory with an accuracy of $\Delta \mathrm{X}_{\text {screen }}= \pm 500 \mu \mathrm{m}$ in the vertical direction. Such information is especially relevant to monitor the beam trajectory at $e^{-}$beam injection, during which the amplitudes of the photon beam trajectory oscillations are expected to be larger than under standard conditions $(<50 \mu \mathrm{m})$. A water-cooled photon cup was manufactured and placed at the BIA, immediately before B-FLUO, as depicted in Fig. 2. A 3D model of the device can be seen in Fig. 4(b). Its aim is to collect and absorb those photons that make their way through the test bench to its back end before impinging on B-FLUO. The photon cup, manufactured from an OFHC copper bulk, has a concave conical shape with a vertex angle of $40^{\circ}$ in order to avoid backreflection of photons. Its outer diameter is $80 \mathrm{~mm}$, having an area a factor of 4 larger than the nominal diameter of the samples, so that the maximum number of photons can be intercepted. The photon cup can be pneumatically actuated in the vertical direction in order to set aside the beam trajectory and allow the photons to reach the B-FLUO screen when required. The photon cup is also equipped with an insulated electrode on which a positive bias voltage can be applied so as to measure the photoelectrons generated at the photon cup due to photon irradiation. The reading of such an electrode provides especially relevant information regarding the number of photons reaching the back end of the test bench.

\section{DIAGNOSTICS}

\section{A. PSD}

In order to perform PSD studies, the BAGs of the BESTEX are strategically positioned along the setup. The positions of front and back BAGs are symmetric with respect to the middle point of the bench, in such a way that the conductance to the center of the sample is similar for both gauges. Such conductance, which depends on the geometry and molecular transmission probability [12] of each sample, was calculated for $\mathrm{H}_{2}$ to be $18 \mathrm{l} / \mathrm{s}$ in the simplified case of a tube of $40 \mathrm{~mm}$ diameter at room temperature centered at the SCA. It is worth noting that, even though the temperature of the real FCC-hh BS can be resembled at the sample, the BESTEX does not count with a surrounding $1.9 \mathrm{~K}$ cold bore around it. Nevertheless, the well-determined geometries and conductances along the setup allow one to precisely determine the molecular yields of the surface of each sample in the absence of the pumping effect of the $1.9 \mathrm{~K}$ cold bore. A cylindrical chimney is mounted at the middle point of the test bench, which faces a circular vacuum port at the center of the sample (see Fig. 5). The chimney separates the SCA from the MIA as depicted in Fig. 2. Its aim is to allow the transport of molecules from the inner part of the sample towards the MIA. This geometry allows the M-BAG and RGA to perform analyses of the residual gas species and measure photon desorption yields from the inner part of the sample under study. The RGA was calibrated in situ [13] against M-BAG by injecting $\mathrm{H}_{2}, \mathrm{CO}, \mathrm{CO}_{2}$, and $\mathrm{CH}_{4}$ into the system.

In order to thermally isolate the sample and avoid heat transfer between the sample and the rest of the setup, the chimney and the sample remain out of contact. This results in a nonperfectly leak-tight transition which enables the

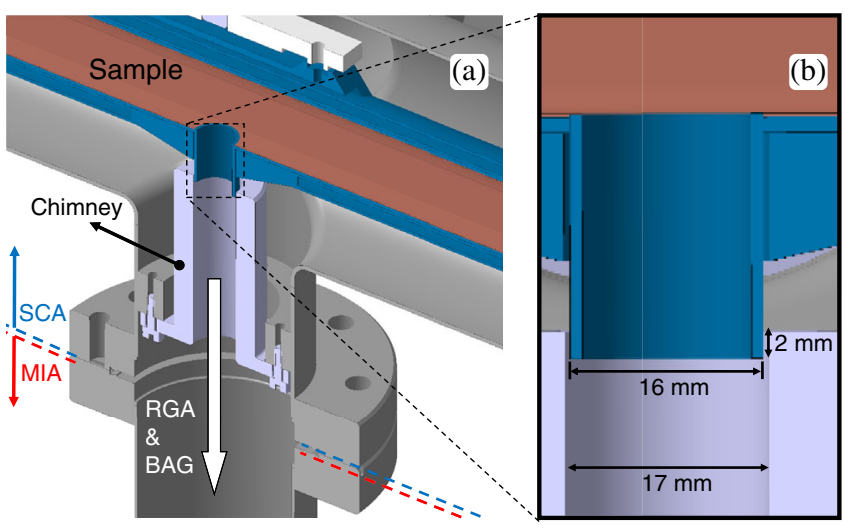

FIG. 5. Descriptive 3D model of the chimney connection, between the MIA and the inner part of the sample under study at the BESTEX. 
escape of a residual number of molecules towards the SCA while traveling through the chimney from the inner part of the sample to the MIA. In order to minimize the effect of such parasitic pumping [14], the distance between the chimney and the sample was minimized, taking into account the available mechanical tolerances. As graphically presented in Fig. 5(b), the vacuum port on the sample (outer diameter $16 \mathrm{~mm}$ ) intrudes $2 \mathrm{~mm}$ inside the chimney (inner diameter $17 \mathrm{~mm}$ ). Such geometry entails the presence of a ring-shaped gap of $26 \mathrm{~mm}^{2}$. According to such geometry, MOLFLOW [15] software was used to estimate that less than $8 \%$ of those molecules originating at the samples inner walls will be residually pumped towards the SCA vessel while traveling though the chimney.

\section{B. Heat loads}

The BESTEX is equipped with 12 PT100 temperature sensors located along the sample under study. Their calibration curve is given in Ref. [16]. The wires of the temperature sensors are connected to a total of three subD25 electrical feedthroughs which bring the signals outside of the vacuum chamber. Each of the feedthroughs is placed in one of the DN63 vacuum ports above described. LakeShore Cryotonics Quad-Twist UHV compatible wires were used to connect the temperature sensors to the vacuum side of the feedthroughs. The wires are formed by four leads of $0.127 \mathrm{~mm}$ diameter phosphor bronze wire twisted by pairs. Each lead is electrically insulated with a UHV compatible thermoplastic resin [17]. One twisted pair is used for sensor excitation and the other twisted pair for sensor output voltage in order to minimize pickup of electromagnetic noise by induced currents through the leads.

\section{Photon reflectivity effects}

After first irradiation on the sample's inner walls, photons internally reflected towards the back end of the sample are collected at the photon cup. The photoelectron signal collected at its isolated electrode is mainly directly related to the number of photons that impinge on it. A PXIe-4135 unit from National Instruments was utilized as an electrometer (sensitivity of $10 \mathrm{fA}$ ) and bias voltage source, able to polarize the electrode at voltages in the -200 to $+200 \mathrm{~V}$ range. According to the geometry of the photon cup, described in Sec. II, photons impinging on it would need to undergo a minimum of one reflection, after incidence at an angle of around $40^{\circ}$, in order to make their way back to the photon cup's electrode. The latter is located $60 \mathrm{~mm}$ away from the BS axis, where photon irradiation takes place. Under such conditions, according to tabulated reflectivity data [18], the signal due to the impingement of photons (after reflection in the photon cup) in the photon cup's electrode is assumed to be negligible. In order to perform reflectivity studies, the setup is positioned in two different geometric configurations, namely, straightthrough and in-sample irradiation. The current measured at
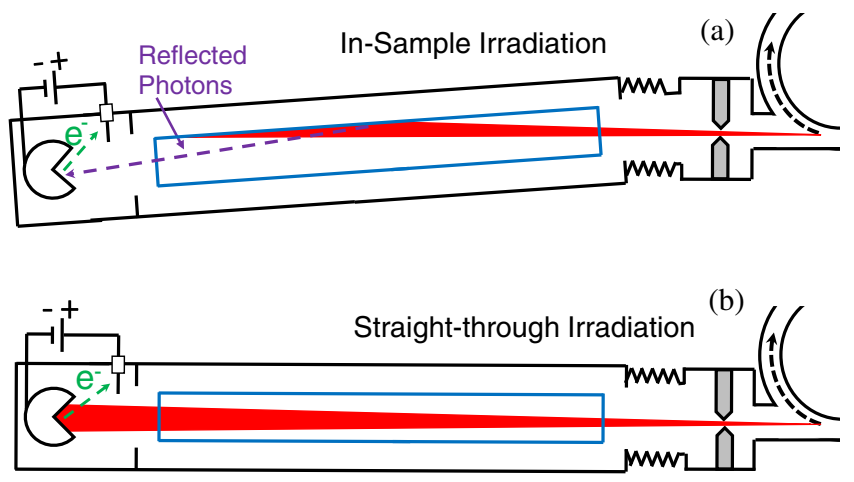

FIG. 6. Schematic description of the operation principle of the reflectivity studies at the BESTEX. Photoelectrons originating at the photon cup (green arrow) are collected by the electrode's positive bias and measured. (a) In-sample irradiation geometry. The photon cup is irradiated by photons reflected on the sample (purple arrow). (b) Straight-through irradiation geometry. The photon cup is irradiated by direct SR from KARA's bending magnet.

the photon absorber's electrode at each configuration are $I_{\text {refl }}$ and $I_{\text {straight }}$, respectively. Such values can be compared to obtain the fraction $F=I_{\text {refl }} / I_{\text {straight }}$. A schematic description of each configuration is presented in Fig. 6. At this point, it must be taken into account that the photoelectron yield of the photon cup's surface, and, hence, $I_{\text {refl }}$ and $I_{\text {straight }}$, depend not only on the number of photons reaching the photon cup, but also on the energy spectrum of the photons reaching it. In this regard, the energy spectrum of the photons reaching the photon cup in the straightthrough geometry strongly differs from those of in-sample geometry due to the reflection to which the latter are subjected [18]. Keeping this in mind, the value of $R$ is useful to qualitatively compare different design candidates of the FCC-hh BS for which different strategies have been considered concerning the interception scheme of the impinging SR [10]. Hence, quantitative x-ray reflectivity cannot be directly measured at the BESTEX. Nevertheless, the data acquired at the BESTEX represent an extremely relevant input to benchmark simulation techniques. More importantly, such data, complemented with specific reflectivity measurements of small samples [19], will allow us to carry out the real machine's performance calculations.

\section{Photoelectron generation}

Samples at the BESTEX can be equipped with the necessary gauges to perform measurements related to the SR-based effects during sample irradiation. This work presents the results obtained on a sample (described in detail in Sec. V) that was equipped with a longitudinal clearing electrode deposited via cold-spray techniques [20] along the inner walls on the sample. This electrode is an extra gauge exceptionally added to one of the samples to carry out specific experiments at the BESTEX. No clearing electrode 
installation is foreseen for the baseline of the FCC-hh BS. The PXIe-4135 unit was used to measure the photoelectron current on the clearing electrode while varying the bias voltage applied to it. The electrode was positioned in a region free of direct photon irradiation. A ceramic layer was deposited between the BS inner wall and the clearing electrode in order to provide electrical insulation.

By applying a positive bias to the clearing electrode, the electron population inside the sample during irradiation can be explored. In this respect, the BESTEX has three great advantages: (i) The length of the samples allows one to confidentially explore photoelectron emission at different angles of incidence (down to $2 \mathrm{mrad}$ ), (ii) the high SR flux allowed into the BESTEX allows one to study the evolution of surface conditioning on the photoelectron emission, and (iii) the high critical energy of KARA allows one to perform measurements at critical energies yet unexplored.

Because of the sample geometry here presented (closed tubular shape as shown in Sec. V), a positive bias at the clearing electrode will collect not only the photoelectrons generated due to sample irradiation, but also the secondary electrons generated at the sample's inner walls by the primary photoelectrons. Discernment of both kinds is not possible in this case. However, the electron population inside the sample will always be subjected to the photoelectron yield (PEY) of the sample inner walls. Dedicated sample shapes for quantitative PEY analysis are planned to be manufactured and measured at the BESTEX. Such samples imply an open geometry and electrical insulation in order to directly measure photoelectron currents. So far, simplified tubular samples have been used to commission the setup. Yet the data obtained at the BESTEX's clearing electrode in a closed tubular geometry represent an extremely valuable input for electron cloud (EC) simulation tools [21-24] to render a close-to-reality scenario of the real machine. By taking also into account secondary electron yields of the sample's inner wall surfaces as well as the effect of the magnetic and electric fields to which photoelectrons are subjected, accurate EC foresight at the FCChh can be carried out.

\section{ALIGNMENT}

Before the installation of the BESTEX at KARA, the positions of the collimator and the sample under study, housed at the front end and SCA, respectively, were defined with respect to the fiducials located at the outer part of each vacuum chamber. In this way, their location after setup assembly can be derived from the position of the fiducials. The fiducialization was performed at the metrology lab of CERN by using a commercial Prismo Ultra machine from ZEISS. The accuracy of the measurement was $\Delta \mathrm{X}_{m}=$ $\pm 10 \mu \mathrm{m}$. During the installation of the BESTEX in the KARA storage ring, the position of each fiducial with respect to the SR source at the bending magnet was monitored by using a Leica AT401 absolute laser tracker.
The ideal position of each fiducial was calculated in order to place each chamber in their designated orientation. Such an orientation was chosen for the collimator and the sample under study, installed in their inner part, to be aligned with respect to KARA's electron beam orbit. The alignment procedure during installation of the BESTEX was carried out so that the position of each fiducial, measured with the laser tracker, is coincident with its ideal position with an accuracy of $\Delta \mathrm{X}_{\mathrm{fidu}}= \pm 50 \mu \mathrm{m}$.

After the BESTEX was pumped down and brought to UHV conditions, the setup alignment was validated by making use of the incoming SR. To this end, the setup was set in the straight-through configuration, so as to directly irradiate the photon collector. The slits were set to a vertical aperture of $200 \pm 50 \mu \mathrm{m}$. The photoelectron current measured during irradiation of the photon cup was monitored while the position of the collimator's gap was modified in the vertical direction. The whole SR flux profile was then scanned, in such a way that the photon power and flux allowed into the test bench find their maximum when the position of the collimator's gap center is coincident with KARA's $e^{-}$beam plane. In this configuration, the photoelectron current measured at the electrode shall be maximum as well. The values of the SR flux and power were calculated for different positions along the vertical direction. Figure 7(a) shows the calculated SR flux and power profiles, as extracted from the crotch absorber, represented as a function of the height of each position with respect to the $e^{-}$ beam plane (D). For both curves, the maximum of each profile is coincident with $\mathrm{D}=0$. Figure 7(b) shows the photoelectron current measured at different positions of the collimator's gap center as a function of D. The size of the

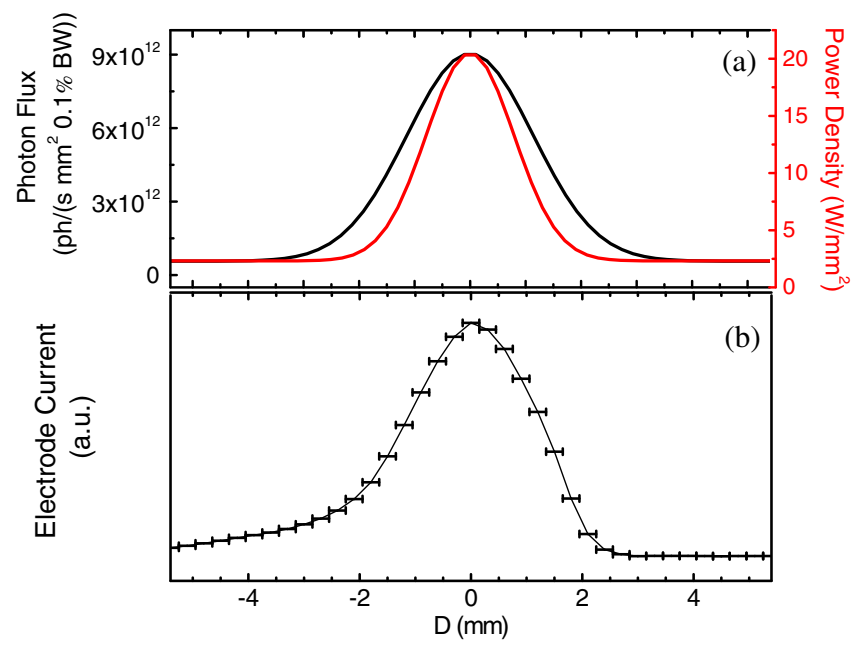

FIG. 7. (a) Calculation of the distribution of photon flux (black line) and power (red line) along the vertical direction as extracted from KARA's bending magnet. (b) Signal of the current measured at the photon cup's electrode as a function of the collimator's position. Both plots are referenced to KARA's electron beam orbit $(\mathrm{D}=0)$. 
horizontal error bars is $300 \mu \mathrm{m}$, in agreement with the collimator's vertical gap size and positioning accuracy. It can be observed that the evolution with $\mathrm{D}$ of the measured photoelectron current closely resembles the photon flux and power distribution depicted in Fig. 7(a). Moreover, the photoelectron current signal finds its maximum at $\mathrm{D}=0$. This confirms that the alignment and the fiducialization processes were satisfactorily performed, allowing one to position the equipment located at the inner part of the setup with respect to the $e^{-}$beam plane with an accuracy of at least $\Delta \mathrm{X}_{\text {align }}= \pm 150 \mu \mathrm{m}$. The trajectory of the incoming SR beam was benchmarked by analyzing the information of both F- and B-FLUO for different collimator apertures. Both fluorescent screens were as well subjected to fiducialization so that the position of the grid lines with respect to the $e^{-}$ beam orbit is known. After visual inspection of the SR footprint, taking into account the distance between both fluorescent screens and the size of their reference grids, the trajectory of the photon beam was confirmed to be parallel to the sample's equatorial plane with an error $\Delta \theta_{\mathrm{ph}}= \pm 1 \mathrm{mrad}$.

\section{THE SAMPLES}

The BESTEX was conceived to perform studies on FCC-hh BS prototypes. As described elsewhere [6,10], the design of the FCC-hh BS implements a main chamber (MC) and an antechamber (AC) separated by a shield plate. A vertical slot aperture, located at the BS's equatorial plane, allows the SR to travel from the MC to the AC and impinge at its inner walls. In order to optimize the SR coping efficiency of the FCC-hh BS, several geometries have been explored, each of them implementing different slot sizes as well as SR interaction features at the AC's inner walls. For the commissioning campaign of the BESTEX, two FCC-hh BS prototypes, BS1 and BS2, were manufactured in faithful compliance with an actual FCC-hh BS design. A descriptive cross section of such a design is presented in Fig. 8(a). The slot aperture in this case is $5 \mathrm{~mm}$ high. The AC's inner walls implement a stainless steel photon reflector tip, whose functionality and description are out of the scope of this paper. In agreement with the FCC-hh normal operation scenario [6], the collimator's opening at the BESTEX is set to allow a 2-mm-high SR beam to travel through the $5 \mathrm{~mm}$ BS prototype's slot. The propagation of the errors assumed at the collimator and the sample position (see Sec. IV) defines the accuracy with which this irradiation geometry can be achieved to be $\Delta \mathrm{X}_{\mathrm{SR}}= \pm 200 \mu \mathrm{m}$. It is, however, necessary, in order to ensure that the irradiation takes place at the designated region, to take into account the mechanical tolerances of the sample's manufacturing process and the spatial uncertainties derived from them. More specifically, according to the accepted linearity of the shield plate edges, the samples were assembled assuming a maximum error of $\Delta X_{\text {slot }}= \pm 250 \mu \mathrm{m}$ for the slot's vertical size. As graphically explained in Fig. 8(b), the distance that ideally remains between the SR beam and the slot's limits is

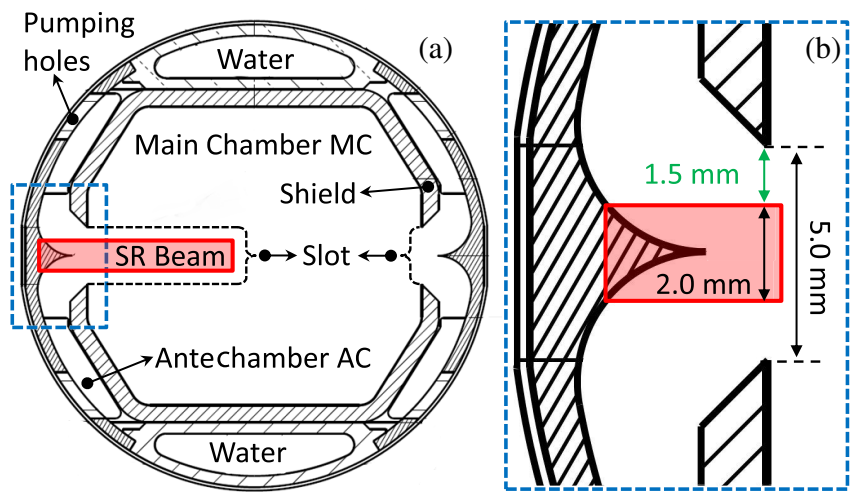

FIG. 8. (a) Cross section of the FCC-hh BS design in which analogy the samples presented in this work were manufactured. The blue dashed square represents the portion of the cross section presented in (b). The red square represents the area of the SR beam. (b) Magnification of the sample's slot aperture of the FCC-hh BS. The red square represents the area of the SR beam.

$1.5 \mathrm{~mm}$, which is larger than the uncertainties derived from sample manufacturing and SR aiming limitations, i.e., $\Delta \mathrm{X}_{\mathrm{SR}}+\Delta \mathrm{X}_{\text {slot }}<1.5 \mathrm{~mm}$. This allows the BESTEX to confidentially direct the SR to the AC across the sample's slot without irradiating the main chamber's shield plate.

The sample counts with two cooling pipes located at the upper and lower part of the sample, respectively. The coolant's inlet (connected to the upper pipe) and outlet (connected to the lower pipe) are at the same extremity of the sample. At the other extremity, a loop allows the coolant to continue flowing from the upper to the lower pipe.

Distributed along the samples, several pumping holes allow the escape of the desorbed molecules, which in the case of the FCC-hh would eventually be pumped by the $1.9 \mathrm{~K}$ superconducting magnet cold bore $[4,6]$ located at its outer part.

The unique difference between BS1 and BS2 is that the latter counts with a longitudinal clearing electrode (see Sec. III D), located at the MC's upper inner wall, deposited via cold-spray techniques [20]. The electrode, $1.8 \mathrm{~m}$ long and $5 \mathrm{~mm}$ wide, collects electrons from all along the sample, and, as explained in Sec. VI, its signal is not affected by electrons originating from foreign devices such as the photon cup. A ceramic layer was implemented between the BS inner wall and the clearing electrode in order to provide electrical insulation.

\section{FIRST RESULTS}

Before insertion into the BESTEX, the samples were cleaned following standard CERN UHV procedures. Then, after installation, bakeout cycles of $24 \mathrm{~h}$ at $150^{\circ} \mathrm{C}$ were performed in order to remain within vacuum pressure limits required to operate the storage ring KARA. After bakeout, the RGA spectrum showed that the gas composition is dominated by $\mathrm{H}_{2}$, indicating an efficient $\mathrm{H}_{2} \mathrm{O}$ removal and 
the absence of vacuum leaks. The vacuum beam pipes of the FCC-hh, as well as those of the LHC, will not be subjected to bakeout processes; for this reason, it is important to note the limitations of baked samples to resemble real conditions of the FCC-hh. This is specially true for PSD results, since molecular yields are known to be affected by the surface chemical changes that take place when exposed to baking temperatures. During the acquisition of the data here presented, the samples were refrigerated by using water as a coolant. Cooling down tests of unbaked samples at cryogenic temperatures have also been carried out in the absence of SR. In such a case, liquid nitrogen was introduced through the same sample's cooling pipes used to refrigerate with water. The tests showed a satisfactory vacuum and mechanical performance, confirming the availability of data acquisition at $77 \mathrm{~K}$. Moreover, due to the intrinsic pumping effect and reduction of thermal outgassing inherent to cold surfaces, the background pressure of the system during cooling down was reduced to values below the safety limits of KARA, ensuring the possibility of measuring unbaked samples at cryogenic temperatures. Tests with SR at liquid nitrogen temperatures are planned in the following years with different samples.

The static pressure (free of photon irradiation) readings of the BESTEX measured at F-BAG and B-BAG in the presence of BS1 are $1.3 \times 10^{-10}$ and $1.7 \times 10^{-10}$ mbar, respectively. The relative discrepancy between both readings remains within the limits of the BAG accuracy (15\%) [25], indicating a symmetric longitudinal pressure distribution along the setup. On the other hand, the static pressure measured through the chimney is $5.7 \times 10^{-10}$ mbar, $\sim 4$ times higher than at the extremities of the setup. Such an effect is due to the limited pumping speed inherent to the setup geometry at the chimney connection and the limited conductance of the sample's geometry.

The plots presented in Fig. 9(a) show the values of the beam energy and beam current during a $24 \mathrm{~h}$ working cycle of KARA. They are presented together with the evolution of the pressure measured by each of the BAGs of the setup [Fig. 9(b)]. During the sample irradiation, the slits which collimate the incoming photon beam were set to have an opening of $2 \times 20 \mathrm{~mm}$ along the vertical and horizontal direction, respectively, cropping $83 \%$ of the total flux and $69 \%$ of the total power delivered from KARA's bending magnet through the crotch absorber. This geometry allows one to resemble the SR parameters of the FCC-hh as presented in Table I. When setting the collimator opening to achieve such values of SR flux and power, the irradiation angle was constrained to $18 \mathrm{mrad}$ versus the $1.35 \mathrm{mrad}$ of the FCC-hh. Such a configuration allowed us to achieve a signal, at each of the measuring devices of the BESTEX, high enough to confidentially commission their functionality. When the FCC-hh's SR glancing angle needs to be resembled, the collimator's opening needs then to be constrained in the horizontal direction. By doing so, a

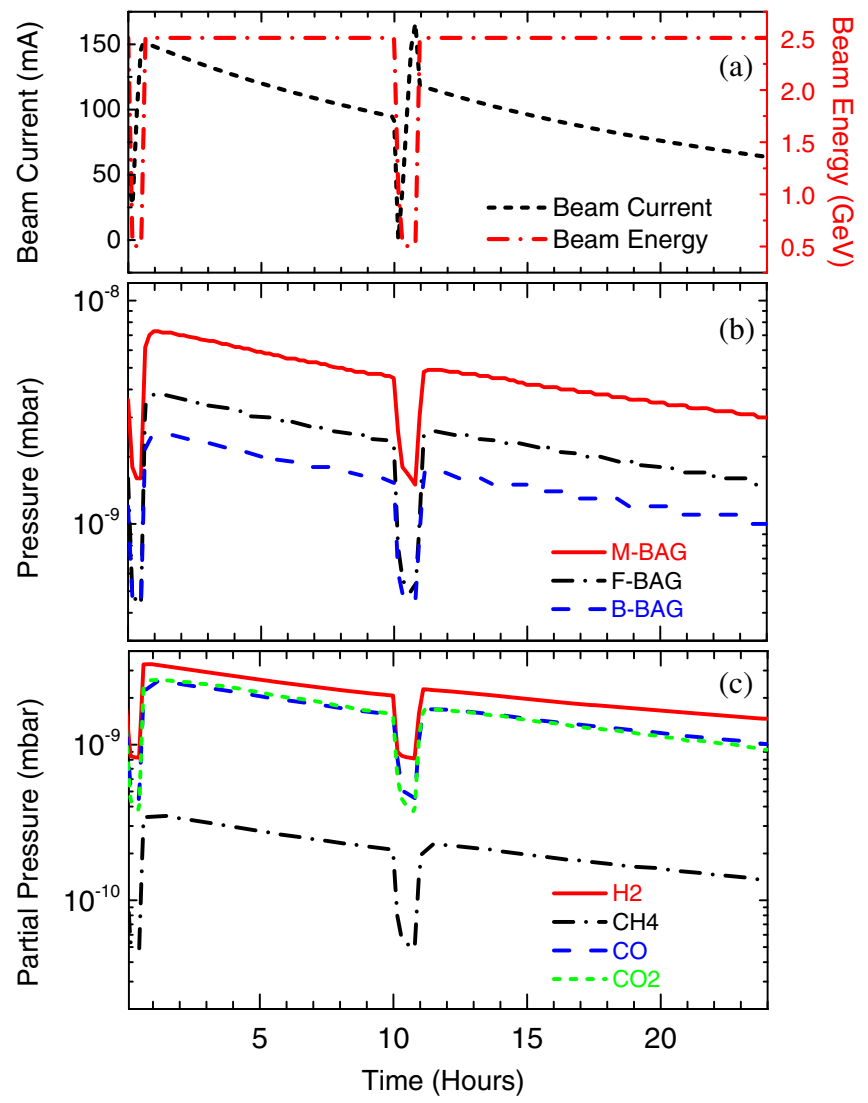

FIG. 9. Evolution as a function of time, during a $24 \mathrm{~h}$ KARA working cycle of (a) beam energy (red line) and beam current (black line), (b) pressure measured at the M-BAG (red line), F-BAG (black line), and B-BAG (blue line), and (c) partial pressures of $\mathrm{H}_{2}$ (red line), $\mathrm{CH}_{4}$ (black line), $\mathrm{CO}$ (blue line), and $\mathrm{CO}_{2}$ (green line).

SR flux and power lower than the nominal of the FCC-hh is allowed onto the sample. This is assumed not to imply a relevant setback to determine the molecular yields of the studied samples, since PSD is mostly dependant on (i) the SR dose, which can be accumulated at different irradiation times for each photon flux, and (ii) the SR critical energy, which remains invariant when horizontally cropping the photon beam. It can be observed that, in good agreement with the values of the static pressure, the pressure measured at the M-BAG is considerably higher than for the front and back extremities. However, unlike

TABLE I. Comparison of the BESTEX (for the configuration of this specific work) and the FCC-hh relevant baseline parameters.

\begin{tabular}{lcc}
\hline \hline & BESTEX & FCC-hh \\
\hline Critical energy [keV] & 6.2 & 4.3 \\
SR flux [ph/s/m] & $4.84 \times 10^{16}$ & $1.7 \times 10^{17}$ \\
SR power [W/m] & 32 & $32^{\mathrm{b}}$ \\
Glancing angle [mrad] & 18 & 1.35 \\
\hline \hline
\end{tabular}

${ }^{\mathrm{a}}$ Power received at the $\mathrm{BS}$.

${ }^{\mathrm{b}}$ Average value. Power ranges between 21 and $42 \mathrm{~W} / \mathrm{m}$. 
TABLE II. Residual gas composition of the BESTEX after bakeout.

\begin{tabular}{lcccc}
\hline \hline & $\mathrm{H}_{2} \%$ & $\mathrm{CH}_{4} \%$ & $\mathrm{CO} \%$ & $\mathrm{CO}_{2} \%$ \\
\hline Static & 92 & 5 & 2 & 1 \\
Dynamic $(2.5 \mathrm{GeV})$ & 43 & 5 & 27 & 25 \\
\hline \hline
\end{tabular}

the static case, the pressure measured at the F-BAG is $60 \%$ higher than for the B-BAG. This effect is ascribed to the outgassing originated at the photon beam collimator which, due to its proximity to the F-BAG, contributes to the pressure reading. The partial pressures of $\mathrm{H}_{2}, \mathrm{CH}_{4}, \mathrm{CO}$, and $\mathrm{CO}_{2}$ measured at the RGA are presented in Fig. 9(c). The results show, in good agreement with previously published results [26,27], that during irradiation of a baked surface $\mathrm{H}_{2}$ is the most abundant gas, followed by $\mathrm{CO}$ and $\mathrm{CO}_{2}$. Table II show the percentage of each gas for the two cases during irradiation of the sample and in the static case.

Figures 10(a) and 11(a) show the values of the beam energy and current during two different $24 \mathrm{~h}$ working cycles of KARA. They are presented together with the current measured at the photon cup electrode [Figs. 10(b) and 10(c)] and the sample's clearing electrode [Figs. 11(b) and 11(c)], respectively. The polarity of the current has been defined as positive when electrons are emitted from the electrode's

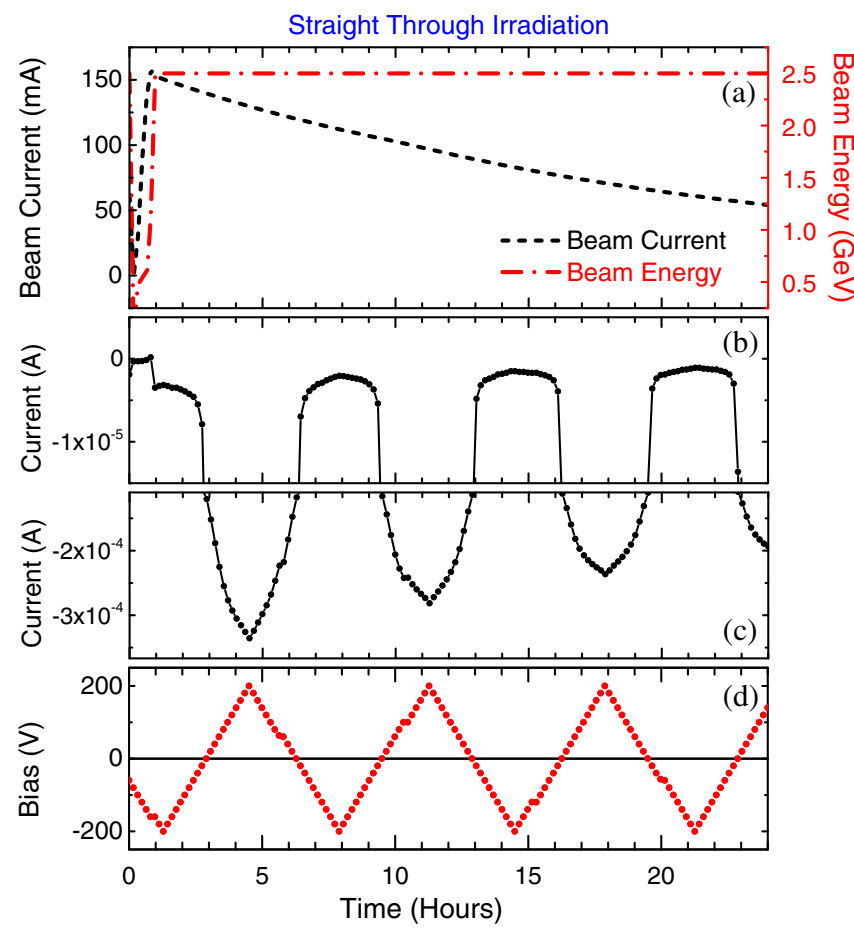

FIG. 10. Evolution as a function of time, during a $24 \mathrm{~h}$ KARA working cycle and irradiation in the straight-through configuration of (a) beam energy (red line) and beam current (black line), (b),(c) current measured at the photon cup's electrode, at different applied bias voltages (positive and negative, respectively), and (d) bias voltage applied to the photon cup's electrode. surface by traveling through the electrometer from the ground. During photon cup electrode measurements, the setup was positioned to perform straight-through irradiation. For the sample's clearing electrode measurements, the setup was positioned to irradiate at in-sample geometry. The bias voltage applied to each electrode was changed between -200 and $200 \mathrm{~V}$ in steps of $20 \mathrm{~V}$ as shown in the bottom panel in each figure. It can be observed in both cases how the measured current increases and decreases following the bias voltage trend. The intensity of the photoelectron current produced in each case decreases as KARA's electron beam current decays with time, indicating a good correlation between the beam current and photon flux.

Figure 10(b) shows the current measured at the photon cup's electrode for negative bias voltages during irradiation at the straight-through configuration. In this case, the intensity remains always below $1 \times 10^{-5} \mathrm{~A}$. During the first hour of the KARA working cycle, before injection of the $e^{-}$beam in the storage ring, a current of $\sim 10^{-9}$ A was measured. Such a value corresponds to the background level of the device in the absence of photon irradiation. A sudden step at $\sim 1 \mathrm{~h}$ is observed during the injection of the $e^{-}$beam at KARA in which the photoelectron current increases to a value of $-4 \times 10^{-6} \mathrm{~A}$. The fact that the measured current is negative at negative voltages suggests that in such a case the measured signal is dominated by photoelectrons generated at the photon cup, of energy high enough to overcome the effect of the bias voltage applied at the electrode (varying from -200 to $200 \mathrm{eV}$ ). The latter is in good agreement with the fact that, as stated in Sec. III C, the number of photons reflected backwards after impingement at the photon cup is negligible.

At positive bias voltages, as shown in Fig. 10(c), the absolute value of the photoelectron current drastically increases, reaching values 2 orders of magnitude higher than those measured at negative voltages. Such a signal corresponds to the photoelectrons generated at the photon cup and attracted by the negative voltage applied to the electrode. Hence, at a given positive bias, the signal is proportional to the flux and energy distribution of the photons arriving to the photon cup.

In the case of the sample's clearing electrode, as shown in Fig. 11(b), the current measured before $e^{-}$beam injection at KARA ( $\mathrm{t} \sim 1$ and $10 \mathrm{~h}$ ) is on the order of $\sim 10^{-9} \mathrm{~A}$. Such a result is in good agreement with the absence of photons allowed into the setup as observed at the photon cup's electrode. After $e^{-}$beam injection at KARA, a drastic increase in the absolute value of the clearing electrode's current, up to $-7.5 \times 10^{-4} \mathrm{~A}$, can be observed for positive bias voltages [see Fig. 11(c)], corresponding to the photoelectron generation after SR impingement at the AC's inner walls.

In order to properly understand the signal from the clearing electrode, it is necessary to ensure that such a signal corresponds uniquely to photoelectrons originating 


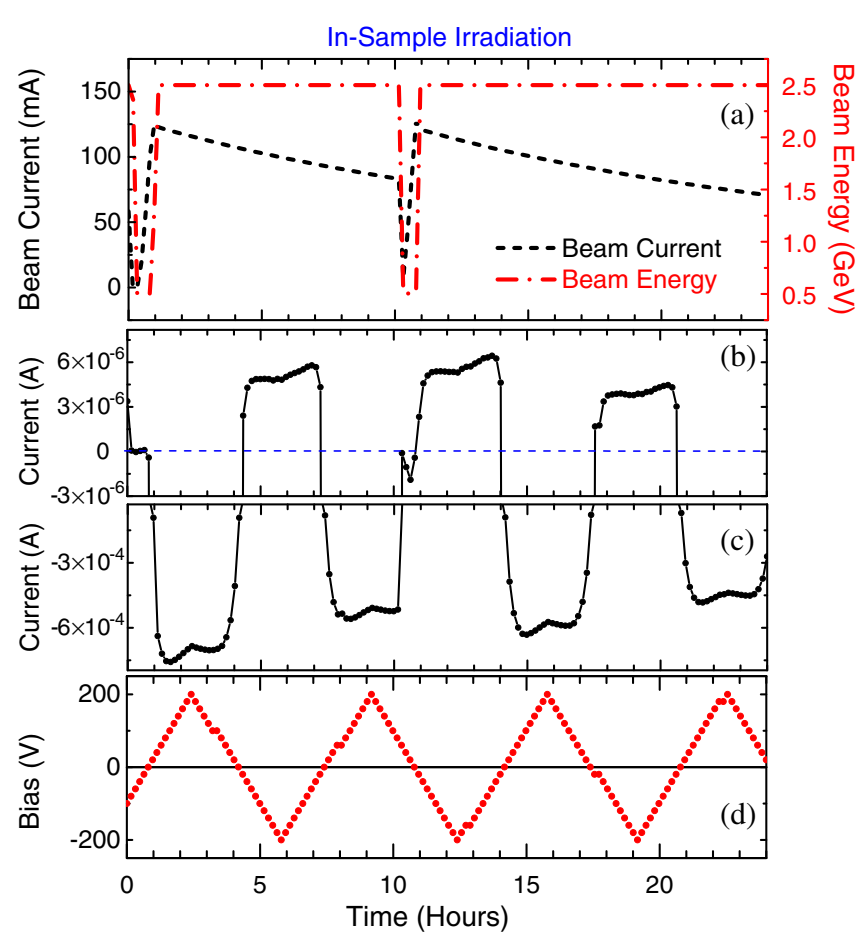

FIG. 11. Evolution as a function of time, during a $24 \mathrm{~h}$ KARA working cycle and irradiation in the in-sample configuration of (a) beam energy (red line) and beam current (black line), (b),(c) current measured at the clearing electrode, at different applied bias voltages (positive and negative, respectively), and (d) bias voltage applied to the clearing electrode.

in the sample and there is no contribution from the photoelectrons originating in the photon cup (after reflection in the sample). To such end, for a short period of a normal working cycle of KARA, the photon cup was momentarily displaced away from the path of the SR beam, allowing the photons (reflected in the sample) to freely travel towards the back fluorescent screen at the very end of the BIA. By observing the signal obtained at the clearing electrode during such a process, the contribution to the clearing electrode's signal of the photoelectrons originating in the photon cup can be observed. A bias voltage of $200 \mathrm{~V}$ was applied to the clearing electrode in order to set a worsecase scenario in which the ability of the photoelectrons, originating at the photon cup, to reach the clearing electrode is enhanced. Figures 12(b) and 12(c) show the signal measured at the clearing electrode and the position of the photon cup (1, at the photon trajectory and 0 , aside the photon trajectory), respectively. Figure 12(a) shows the beam parameters during the measurements. The absolute value of the signal at the clearing electrode shows a general decreasing trend, in agreement with the decrease of KARA's $e^{-}$beam current. No clear response to the change in position of the photon cup is observed. Such facts suggest that the contribution from the photoelectrons generated at the photon cup to the clearing electrode reading can be neglected.
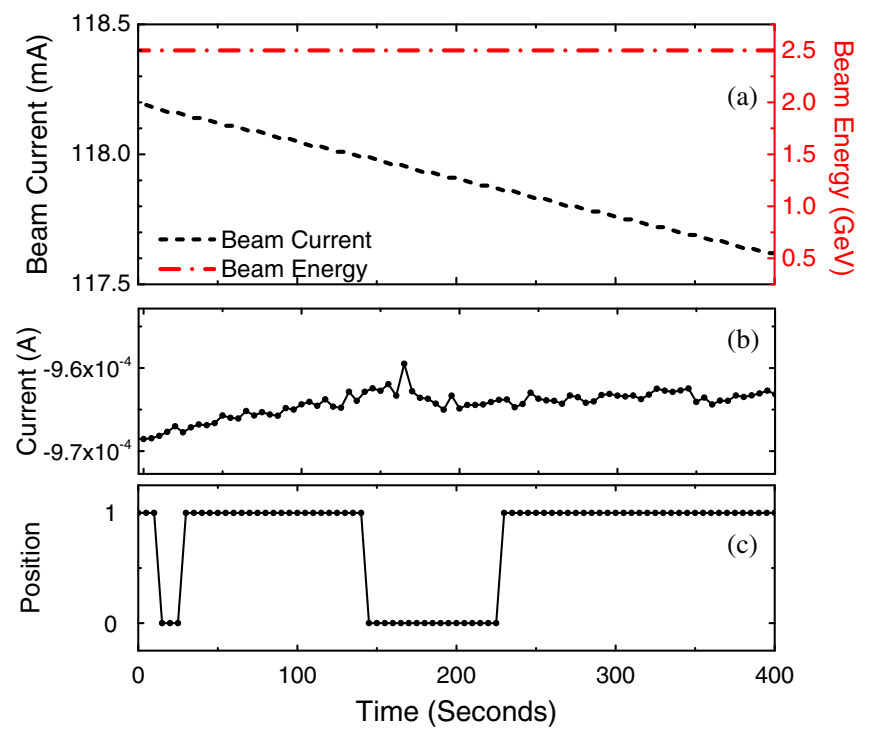

FIG. 12. Evolution of the clearing electrode signal at an applied bias voltage of $200 \mathrm{~V}$ while changing the position of the photon cup, as a function of time during $400 \mathrm{~s}$ of KARA's $e^{-}$beam storage of (a) beam energy (red line) and beam current (black line) and (b),(c) current measured at the clearing electrode. (d) Position of the photon cup (1, at the photon trajectory and 0 , aside the photon trajectory).

On the other hand, the positive currents measured at the clearing electrode for negative bias voltages [Fig. 11(b)] suggest that, despite the contribution of photoelectrons of energy high enough to overcome the bias voltage barrier, the signal is dominated by the photoelectron production at the clearing electrode's surface. According to what is stated in Sec. III C, the contributions from photons reflected in the photon cup to the signal of the clearing electrode have been neglected. Hence, such photoelectrons are assumed to be originated by photons internally reflected towards the inner walls of the sample, before being able to make their way through and exit it. Such information shall be of high relevance to qualitatively compare different FCC-hh BS designs.

Figure 13(a) shows a schematic description of the position of each PT100 installed on BS2. They are installed at the outer part of the sample, along its equatorial plane, in such a way that the SR impinges exactly across the samples walls, keeping the temperature sensors free of direct photon irradiation. The distribution of the power deposited after direct impingement of the SR along the sample was calculated for an $e^{-}$beam energy and current of $2.5 \mathrm{GeV}$ and $75 \mathrm{~mA}$, respectively, by using the software Synrad+ [15] and is shown in Fig. 13(b). The black curve shows an asymmetric profile in which the power density decreases as going forward in the beam direction. Such an effect is ascribed to the intrinsic angular spread of the SR photon fan, due to which, as in the case of the FCC-hh [6], the density of photons reaching the sample decreases as the distance to the bending magnet increases. The red dots represent the 


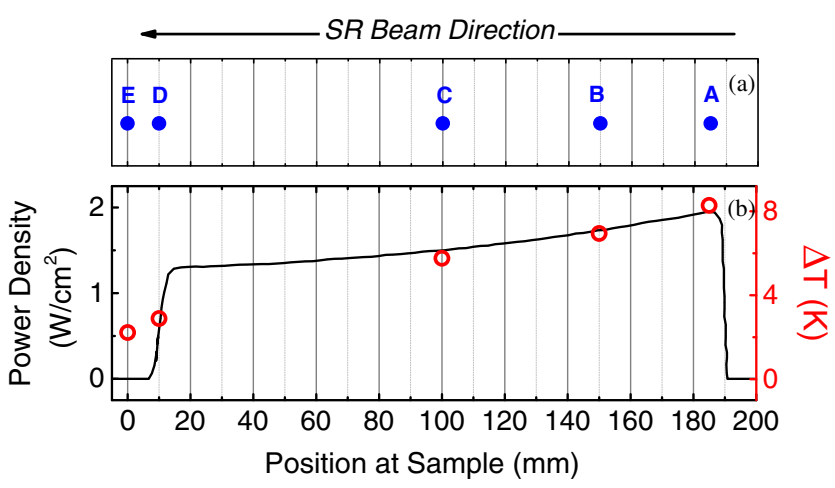

FIG. 13. (a) Schematic description of the position of each PT100. (b) Distribution of the SR power density deposited along the sample calculated by Synrad+ (black line) and temperature increments measured at different points along the sample (red dots) for a $2.5 \mathrm{GeV}$ and $75 \mathrm{~mA} e^{-}$beam.

increments of temperature measured at each position for an irradiation time of $15 \mathrm{~h}$ and $e^{-}$beam energy and current $2.5 \mathrm{GeV}$ and $75 \mathrm{~mA}$, respectively. As expected, the temperature distribution for sensors $\mathrm{A}, \mathrm{B}$, and $\mathrm{C}$ shows a good correlation with the deposited power at each part of the sample. Sensor D is located at the edge of the irradiated section, at a transition region of around $50 \mathrm{~mm}$, where the deposited SR power density drastically decreases from 1.2 to $0 \mathrm{~W} / \mathrm{cm}^{2}$. As a consequence, the temperature measured at sensor D is considerably lower than at A, B, and C. This result indicates that the temperature at the sample resembles the power density distribution. Sensor E is located at the very end of the sample, in a region where no direct photons impinge; however, even though very subtly, it shows a

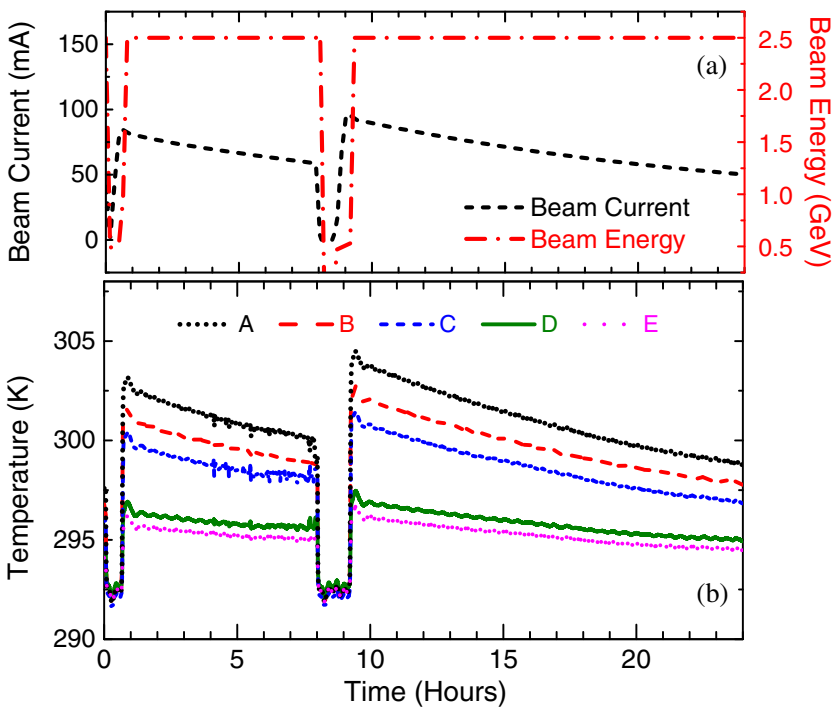

FIG. 14. Evolution as a function of time, during a $24 \mathrm{~h} \mathrm{KARA}$ working cycle, of (a) beam energy (red line) and beam current (black line) and (b) temperatures measured at PT100 A (black line), B (red line), C (blue line), D (green line), and E (pink line). response to KARA's $e^{-}$beam current. This effect has been ascribed to the contribution of photons which, after first impingement on the AC's inner walls, are reflected towards the end of the sample, which contribution is not contemplated in the simulations and hence not represented in Fig. 13(b). The evolution of the temperature measured by each PT100 during a $24 \mathrm{~h}$ working cycle of KARA are presented in Fig. 14(b) together with the corresponding values of the beam energy and current, in Fig. 14(a). The plots show the correlation between the evolution of KARA's $e^{-}$beam current and the temperatures at different points along the sample.

\section{CONCLUSIONS}

A complete description of the BESTEX and its working principle has been presented. The first results obtained during the commissioning period of the BESTEX have shown the capability to perform studies on synchrotronrelated effects on non-leak-tight samples such as the FCC-hh beam screen prototypes. More specifically, the BESTEX allows one to carry out studies related to photon stimulated desorption, photon reflectivity, photon-induced heat loads, and photoelectron generation.

The evolution of the signal acquired at each of the BESTEX's measurement devices has been presented, together with KARA's $e^{-}$beam parameters (beam current and energy) as a function of time for $24 \mathrm{~h}$ working cycles of KARA. The observed pressure profile along the setup during sample irradiation provided an extremely relevant input to take into account during the performance and validation of Monte Carlo simulations. The partial pressures measured across the chimney connection during sample irradiation, in comparison with previous experiments, suggest a satisfactory residual gas analyzer calibration. The temperature along the sample, measured during irradiation, showed a close resemblance with the profile of the power deposited on the sample, even at the edge of irradiated regions. Such an observation suggests that sample temperature monitoring is an efficient way to detect the irradiated regions of the samples under study, as well as to estimate the number of reflected photons reaching sample extremities. The installation of the BESTEX at the KARA storage ring has been described showing that, after the corresponding fiducialization and alignment processes, the setup allows one to mimic the geometric scenario of the FCC-hh, ensuring the irradiation of the samples in the designated region within acceptable margins of error. The experimental results related to photoelectron generation and reflectivity obtained at the BESTEX are an extremely valuable input to benchmark the simulations strategies adopted for the validation of the FCC-hh BS designs. Moreover, manufacturing of dedicated samples will allow the quantitative determination of PEY under relevant conditions for the FCC-hh. A future publication will present the measurements performed at the BESTEX 
during the irradiation of different FCC-hh beam screen prototypes at cryogenic temperatures at the KARA storage ring. Comparison with their corresponding Monte Carlo calculations will also be presented.

\section{ACKNOWLEDGMENTS}

This work was supported by the European Circular Energy-Frontier Collider Study (EuroCirCol) project. EuroCirCol has received funding from the European Union's Horizon 2020 Framework Programme, for research and innovation programme under Grant No. 654305. The information herein only reflects the views of its authors and the European Commission is not responsible for any use that may be made of the information. The authors wish to thank D. Saez de Jauregui, A. W. Grau, N. Glamann and the KARA technician team from KIT as well as B. Henrist and J. Fernandez-Topham from CERN for the valuable technical support. S. Meunier and B. Jenninger from CERN for their support with BAG and RGA calibration. C. Duclos and H. Rambeau for FCC-hh BS protoype manufacturing. A. Gutierrez and J. F. Cortes for control software developement. A. Benoit for the contribution in the developement of BESTEX data acquisition software.

[1] O. Gröbner, Dynamic outgassing, technical report (CERN, Geneva, 1999).

[2] M. Benedikt, B. Goddard, D. Schulte, F. Zimmermann, and M. J. Syphers, in Proceedings of the 6th International Particle Accelerator Conference (IPAC 2015), Richmond, VA, 2015 (JACoW, Geneva, 2015), p. TUPTY062.

[3] M. Benedikt and F. Zimmermann, Towards future circular colliders, J. Korean Phys. Soc. 69, 893 (2016).

[4] M. Benedikt and F. Zimmermann, in Proceedings of the 9th International Particle Accelerator Conference (IPAC 2018), Vancouver, Canada, 2018 (JACoW, Geneva, 2018), pp. 2908-2913.

[5] L. Gonzalez, R. Kersevan, F. Pérez, P. Chiggiato, I. Bellafont, M. Gil Costa, S. Casalbuoni, V. Baglin, C. Garion, and E. Huttel, in Proceedings of the 9th International Particle Accelerator Conference (IPAC 2018), Vancouver, Canada, 2018 (JACoW, Geneva, 2018).

[6] I. Bellafont, M. Morrone, L. Mether, J. Fernandez, R. Kersevan, C. Garion, V. Baglin, P. Chiggiato, and F. Perez, Design of the fcc-hh beam vacuum chamber (to be published).

[7] V. Baglin, 1st WP4 Collaboration Meeting, Barcelona, Spain (2015).
[8] O. Grobner, in Proceedings of the Particle Accelerator Conference, Vancouver, BC, Canada, 1997 (IEEE, New York, 1997), Vol. 3, pp. 3542-3546.

[9] O. Gröbner, Overview of the LHC vacuum system, Vacuum 60, 25 (2001).

[10] F. Perez, in Proceedings of FCC Week19 (plenary), Brussels, Belgium, 2019.

[11] X. Wu, Y. Tao, F. Gao, L. Dong, and Z. Hu, Preparation and photoluminescence of yttrium hydroxide and yttrium oxide doped with europium nanowires, J. Cryst. Growth 277, 643 (2005).

[12] V. Baglin, I. R. Collins, C. Grünhagel, O. Gröbner, and B. Jenninger, First results from COLDEX applicable to the LHC cryogenic vacuum system, technical report, 2000.

[13] O. B. Malyshev and K. J. Middleman, In situ ultrahigh vacuum residual gas analyzer "calibration", J. Vac. Sci. Technol. A 26, 1474 (2008).

[14] V. Baglin, C. Grünhagel, I. R. Collins, O. Gröbner, and B. Jenninger, Synchrotron radiation studies of the LHC dipole beam screen with COLDEX, technical report, 2002.

[15] https://molflow.web.cern.ch.

[16] Platinum RTDs Overview Lake Shore Cryotronics, Inc.

[17] T. J. Nurse and F. Wormwell, The isolation and examination of films from metal surfaces: An improved technique, J. Appl. Chem. 2, 550 (1952).

[18] B. Henke and E. Gullikson, X-Ray Interactions: Photoabsorption, Scattering, Transmission, and Reflection at $E=50-30000 \mathrm{eV}, Z=1-92$, At. Data Nucl. Data Tables 54, 181 (1993).

[19] E. La Francesca, A. Liedl, M. Angelucci, I. Bellafont, L. A. Gonzalez, L. Spalino, F. Siewert, M. G. Sertsu, A. Sokolov, F. Schafers, and R. Cimino (to be published).

[20] C. Garion, in Proceedings of FCC Week18, Amsteram, The Netherlans (2018).

[21] O. Gröbner, Technological problems related to the cold vacuum system of the LHC, Vacuum 47, 591 (1996).

[22] R. Cimino and T. Demma, Electron cloud in accelerators, Int. J. Mod. Phys. A 29, 1430023 (2014).

[23] O. Dominguez and F. Zimmermann, arXiv:1306.5560.

[24] G. Rumolo, F. Ruggiero, and F. Zimmermann, Phys. Rev. Accel. Beams 4, 012801 (2001).

[25] Pressure measurements for the ISR at CERN, edited by J.M. Laurent, C. Benvenuti, F. Scalambrin, and A. Breth (Oesterr Studiengesellschaft fuer Atomenergie $\mathrm{GmbH}$, Austria, 1977).

[26] M. Ady, Monte Carlo simulations of ultra high vacuum and synchrotron radiation for particle accelerators, Ph.D. thesis, CERN, 2016.

[27] O. Gröbner, A. G. Mathewson, and P. C. Marin, Gas desorption from an oxygen free high conductivity copper vacuum chamber by synchrotron radiation photons, J. Vac. Sci. Technol. A 12, 846 (1994). 